\title{
Sexual Addiction Among College Students
}

\author{
Morteza Alibakhshikenari* \\ Department of Medical Sciences and Health Services, Iran
}

*Corresponding author: Morteza Alibakhshikenari, Medical Sciences and Health Services, Tehran, Iran

\begin{abstract}
Previous research suggests that not only is sexual addiction a problem within our society, its prevalence is on the rise Seegers [1]. Exposure to sexually explicit stimuli helps to create this problem. The advent of broadband internet has made this type of material more available than ever before Hall [2]. Pair this alongside other factors that contribute to the development of sexual addiction; childhood abuse and trauma, difficulties in formulating attachments and lack of coping skills and one can see the ease at which this problem can spread. Although the DSM-V has not yet recognized this as an actual disorder, nor included a workable diagnosis, it does appear to be a growing problem. Previous literature by individuals such as; Patrick Carnes and Ariel Goodman has provided us with actual diagnostic criterion that could be utilized to do just that. Evidence supports the fact that the problem does exist and that it is an uncontrollable maladaptive behavior. Our research will help to expand this knowledge by utilizing university students to establish if sexual addiction is presenting within the population we will sample. In determining this we can then move forward with research so as to better understand how and why this may be occurring.
\end{abstract}

\section{Literature Review}

Sexual Addiction affects approximately three to six percent of Americans [1]. According to some this number is thought to be on the rise. Many psychologists place the blame on the massive increase in availability of pornographic materials and sexual stimuli. Paula Hall [2] credits this increase to the advent of commercial broadband. She places this and the advances in cell phone technology as the catalyst in a societal change. This has created a revolution and changed our cultural standards and norms about the appropriateness of sexually provocative stimuli. She argues that as our society changes so to do our perception of what we view a sex addict to be. For the purpose of our upcoming study on sexual addiction I will be reviewing many different articles written about this subject. It is our hope that by examining the various concepts and reviewing studies we can gain better insight as to the prevalence, potential growth, and consequences of this phenomenon.

In one study conducted in 2003, Jennifer A. Seegers took a sample of two hundred and forty university students. Individuals within the sample ranged in age from seventeen to fifty-one. Of the two hundred and forty students, sixty-nine was male and one hundred and seventy were female. Each individual participated on a volunteer basis. Anonymity was assured as each participant's information was strictly confidential. After reviewing the current literature on the subject Seegers hypothesized that although the characteristics and how these manifests differ between men and women, research should show that sexual addiction equally affects both sexes.

To gather data Seeger utilized the Sexual Addiction Screening Tool (SAST; Carnes, 1989) for male participants and the Women's Sexual Addiction Screening Tool (W-SAST; Carnes \& O'Hare, 2000) for women. The SAST is a twenty-five-question survey that can be used to identify individuals possessing behaviors consistent with sexual addiction. For this survey subjects answered yes or no to a multitude of questions relating to sexual addiction. An answer of yes, in thirteen or more questions, indicates that further assessment and treatment may be necessary. The W-SAST is scored differently. A score of six or more is indicative of the subject needing to be further evaluated. What she found was that $17.4 \%$ of the male subjects fell under the category of needing further evaluation and $8.7 \%$ potentially being at risk for sexual addiction. The 
female participant's results were surprisingly higher with $32.2 \%$ needing evaluation and $13.5 \%$ at risk. Although her hypothesis was not supported, with overall only $5 \%$ of males needing further evaluation compared to a staggering $22.9 \%$ of females. Her research plainly shows that the problem not only exist, but effects society far different than previous studies had shown. What was once thought to affect men and women equally can be seen here to drastically lean towards one side.

So, if literature supports the fact that sexual addiction is prevalent and possibly on the rise, what does it says about the cause? Attachment style is said to play a role in men who develop sexual addiction. Zapf, Greiner \& Carrol [3] In their study of fifty-six male addicts, they found that $44 \%$ of those studied exhibited fearfulavoidant characteristics. Twenty-eight percent demonstrated preoccupied attachment style and twenty percent, DismissiveAvoidant Attachment style. This left only eight percent to actually display a healthy secure attachment style. While this may not explain all cases of sexual addiction it does help to show that those individuals who have trouble developing healthy interpersonal relationships are more likely than those who do not, to be at risk.

Another factor that appears to correlate with sexual addiction is sexual abuse during childhood. Griffee, O Keefe, Stroebel, Beard, Swindell and Young [4] They state that, "Childhood Sexual assault is extremely common in the histories of sex addicts." Paula Hall [5] offers two different models which can be used to understand sexual addiction. The first, the BERSC Model, uses a biopsychosocial approach. This model identifies five major influences to which we can tie sexual addiction. These are biological, emotional, relationship, society, and culture. In this model Hull recognizes that these factors not only play a role in determining if an individual develops a sexual addiction, but also influence each another. The OAT model is another model that can be used to help develop objectives and aid a therapist in developing an efficient treatment plan. What the OAT model does, is identify certain areas that the therapist should focus on. That is, are the behaviors part of a pre-existing problem, what triggers them, and how are they maintained? In understanding this, the therapist is better equipped to combat the problem.

What exactly constitutes sexual addiction? When can we say an individual has a pervasive problem? How to we classify this as disorder and what are the diagnostic criteria. Patrick Carnes began asking this question in 1983. He argued that sexual addiction was not only diagnosable but was rooted in uncontrollable sexual behavior. Gold \& Heffner [6] Others argue that sexual addiction is not an actual diagnosable disease. These individuals criticize the utilization of a medically backed disease model in classifying behaviors as a disorder, which can vary from culture to culture. Yet according to Coleman (1992) It is not that these behaviors go against the norm that help to classify it as an addiction, instead it is the negative impact they have upon the lives of the individuals suffering from them that do. So, if we put this all-in perspective
Sexual Addiction is maladaptive compulsive sexual behavior that an individual is unable to refrain from.

Now that we have identified Sexual behavior as a pervasive growing, problem within our society, what is it that we need to recognize in order to identify it? One interesting fact about Sexual Addiction is that many of its symptoms mirror those of other addictions. Shames, guilt, anxiety relief, anger, all of these are emotions that can be found to hold a causal relationship in most addictions. Sexual addiction is no different. Thaddeus Birchard [7] cites Goodman [8] as suggesting that "addiction grows out of impairment in the self-regulatory mechanism of the self." (p.170) He explains that this serves to help the individual cope with painful emotions, experiences and traumas. In their article, Rosenberg, Carnes and O Conner [9] propose actual diagnostic criteria for Sexual Addiction. Ariel Goodman defines Sexual addiction as "a maladaptive pattern of sexual behavior, leading to clinically significant impairment or distress, as manifested by three (or more) of the following" [9].

I. Tolerance, as defined by either of the following:

a) Need for markedly increased amount or intensity of the sexual behavior to achieve the desired effect.

b) Markedly diminished effect with continued involvement in the sexual behavior at the same level of intensity.

II. Withdrawal, as manifested by either of the following:

a) Characteristic psycho-physiological withdrawal syndrome of physiologically described changes and/or psychologically described changes upon discontinuation of the sexual behavior.

b) The same (or a closely related) sexual behavior is engaged in to relieve or avoid withdrawal symptoms.

I. The sexual behavior is often engaged in over a longer period, in greater quantity, or at a higher level of intensity than was intended.

II. There is a persistent desire or unsuccessful efforts to cut down or control the sexual behavior.

III. A great deal of time is spent in activities necessary to prepare for the sexual behavior, to engage in the behavior, or to recover from its effects.

IV. Important social, occupational, or recreational activities are given up or reduced because of the sexual behavior.

Goodman is not the only person to suggest diagnostic criteria for sexual addiction. Patrick Carnes [10,11] presented ten similar criteria in an article published in 1991. While actual diagnostic criterion has yet to be approved for addition in to the Diagnostic Statistical Manual it is clear that literature supports this being a real and present problem. In quantifying Sexual Addiction Carnes helped to advance research within the area. His ten-item questioner, 
the PATHOS and SAST-R have given psychologist some useful tools in helping to identify Sexual Addiction.

\section{Method}

The current study consisted of sixty-five university students at Texas A\&M University-Commerce. The participants were recruited from various FRA classes within the athletic department. Ages of participants ranged from eighteen to forty-four. Forty-eight percent of subjects were male with the remaining fifty-two percent being female. Racial demographics consisted of twenty-six percent of subjects identifying as Caucasian, forty-two percent African American, nineteen percent Hispanic, nine percent Asian, with the other five percent identifying as Native American or other. Twentyeight percent of participants were seniors in college. Twenty-two percent were juniors, and thirteen percent were sophomores. Finally, two percent were classified as freshman. When reporting sexual activity eighty-nine percent identified as being sexually active with only nine percent being virgins. One percent of subjects chose to not answer the question related to sexual activity.

Each participant was asked to complete a short packet containing an informed consent, demographics sheet and the Sexual Addictions Screening Tool revised edition (SAST-R). The SAST-R is an instrument created by Patrick Carnes utilized to identify problematic sexual behaviors that could suggest sexual addiction. It utilizes five categories of items (core, internet, Men's, Women's, and Homosexual Men's) to score participants answers. Once scored these items are categorized once more into four separate dimensions (Preoccupation, Loss of Control, Relationship Disturbance, and Affect Disturbance. A score of two or more in any one dimension indicates a cause for concern in this area and could signify addiction.

\section{Results}

When the results of the survey were calculated it was found that twenty-five percent of individuals in the study answered questions consistent with sexual addiction. Of this twenty-five percent, Affect Disturbance appeared to have the highest number of individual scores. Twenty percent of participants had scores high enough to reach or exceed the cut-off in this dimension. The next highest scoring dimension was that of Preoccupation. Fourteen percent of subjects had scores above the cutoff. The remaining individuals were split between Loss of Control (7.7\%) and Relationship Disturbance (3.1\%). When looking at the subscales the highest scoring area was that of Women's items (16.9\%). Following behind that were Men's items (10.8\%), Internet Items (7.7\%), and Homosexual Men's Items (3.1\%). Those subjects answering yes to Core items were low at $7.7 \%$. What this tells us is, that addictive problems do appear to present within the sample. This problem can mostly be seen in a preoccupation with sexual activities and emotional disturbances in response to this preoccupation. While the size of the sample limits making any real predictions about the prevalence of sexual addiction within the target population, it does show that it can be found. In sample of only sixty-five students we were able to show that twenty-five percent appeared to report some behaviors consistent with sexual addiction. Further research is needed to determine if this trend would continue under a larger sample size.

\section{Conclusion}

Sexual Addiction affects millions of people throughout the world. It disrupts lives and has the potential to tear families apart. Sexual Addiction can find in roots in many places. Childhood Trauma, Difficulties in developing healthy attachments, or simply the excessive availability of sexual and erotic materials in today's digital society. Whatever the roots, Sexual addiction appears to be a pervasive ever-growing problem. Studies such as those mentioned above, help us to gain better insight and understanding as to the breadth of the problem. In a simple survey of sixty-five university students we were able to find that twenty-five percent presented traits and behaviors consistent with sexual addiction. More in-depth research needs to be conducted. It is our hope that we can contribute to this area of study with our research on Sexual Addiction among college students.

\section{References}

1. Seegers JA (2003) The prevalence of sexual addiction symptoms on the college campus. Sexual Addiction \& Compulsivity 10(4): 247-258.

2. Hall P (2013) A New Classification Model for Sex Addiction. Sexual Addiction \& Compulsivity 20(4): 279-291.

3. Zapf JL, Greiner J, Carroll J (2008) Attachment styles and male sex addiction. Sexual Addiction \& Compulsivity 15(2): 158-175.

4. Griffee K, O Keefe SL, Stroebel SS, Beard KW, Swindell S, et al. (2012) On the brink of paradigm change? Evidence for unexpected predictive relationships among sexual addiction, masturbation, sexual experimentation, and revictimization, child sexual abuse, and adult sexual risk. Sexual Addiction \& Compulsivity 19(4): 225-264.

5. Hall P (2014) Sex addiction-an extraordinarily contentious problem. Sexual and Relationship Therapy 29(1): 68-75.

6. Gold SN, Heffner CL (1998) Sexual addiction: Many conceptions, minimal data. Clinical Psychology Review 18(3): 367-381.

7. Birchard T (2011) Sexual addiction and the paraphilias. Sexual Addiction \& Compulsivity 18(3): 157-187.

8. Goodman A (1998) Sexual addiction: An integrated approach. Madison, CT: International Universities Press.

9. Rosenberg KP, Carnes P, O Connor S (2014) Evaluation and treatment of sex addiction. Journal of sex \& marital therapy 40(2): 77-91.

10. Carnes PJ (1991) Don't call it love: Recovering from sexual addiction. New York, NY: Bantam Books.

11. Carnes PJ (1997) Sexual anorexia: Overcoming sexual self-hatred. Center City, MN: Hazelden. 
(c) (i) This work is licensed under Creative

To Submit Your Article Click Here: Submit Article

DOI: 10.32474/LOJMS.2018.02.000142

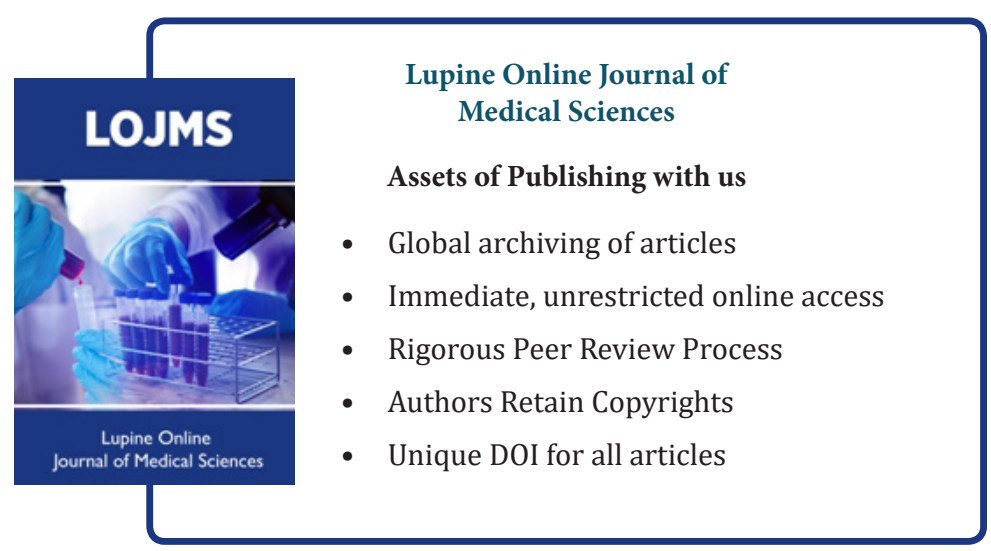

